\title{
LEVANTAMENTO EPIDEMIOLÓGICO DOS DIAGNÓSTICOS HISTOPATOLÓGICOS DE UM CENTRO DE REFERÊNCIA EM PATOLOGIA BUCOMAXILOFACIAL EM UM PERÍODO DE 10 ANOS
}

\author{
Bernadete Dias Neto Estudante do Curso de Odontologia da \\ Escola Bahiana de Medicina e Saúde \\ Pública \\ Alena P. Medrado \\ Doutora em Patologia Humana pela \\ FIOCRUZ/UFBA. Professora Adjunto da \\ Escola Bahiana de Medicina e Saúde \\ Pública \\ Sílvia Regina A. Reis \\ Doutora em Patologia Oral pela \\ Universidade Livre de Berlim. Professora \\ Adjunto da Escola Bahiana de Medicina e \\ Saúde Pública
}

\begin{abstract}
Resumo
O levantamento epidemiológico da casuística das patologias que acometem o complexo bucomaxilofacial é de fundamental importância para a clínica odontológica. Todavia, poucos estudos relacionados a este tema têm sido publicados na literatura, a despeito de sua relevância para a viabilização de programas de saúde. Neste contexto, o objetivo deste trabalho foi avaliar a frequência e os principais tipos de patologias que são comumente biopsiados e enviados ao Serviço de Patologia Bucomaxilofacial do Curso de Odontologia da Escola Bahiana de Medicina e Saúde Pública. Através da realização de um estudo retrospectivo abrangendo os dados coletados durante os anos de 2001 a 2010, foram avaliadas 1095 fichas de biópsias. Considerando o diagnóstico histopatológico de cada caso, avaliou-se a freqüência das lesões patológicas, a distribuição geral das amostras quanto ao sexo e idade, a localização das lesões, e o diagnóstico clínico e histopatológico. As patologias mais freqüentes foram a hiperplasia fibroepitelial (16,62\%); inflamação crônica inespecífica $(6,30 \%)$ e cisto radicular/residual $(4,87 \%)$. A importância de se buscar o correto diagnóstico da patologia apresentada pelo paciente é tão importante quanto a escolha dos métodos e técnicas a serem utilizadas no seu tratamento, ou controle. É evidente que quanto melhor e mais preciso for o diagnóstico, mais eficiente será o tratamento desenvolvido.
\end{abstract}

Palavras-chave: Biópsia; Epidemiologia; Patologia Bucal.

\section{EVALUATION OF PATHOLOGY EXTENSION ACTIVITY OF EBMSP - A TEN YEARS FOLLOW- UP (2001-2009)}

\begin{abstract}
The Epidemiological investigation of the diseases affecting the maxillofacial structures is of fundamental importance to the dental clinic. However, few studies on this subject have been published in the literature, despite its relevance to the viability of health programs. In this context, the objective of this work was to assess the frequency and the main types of diseases that are commonly biopsied and sent to the Pathology Department, School of Dentistry from EBMSP. By conducting a retrospective study covering the data collected during the years 2001 to 2010, 1.095 biopsies were evaluated. Considering the histopathological diagnosis of each case, it was also evaluated the frequency of pathological lesions, the overall distribution of the samples by sex and age, lesion location, and clinical and histopathological diagnosis. The most frequent diagnoses were fibroepithelial hyperplasia (16.62\%), nonspecific chronic inflammation $(6.30 \%)$ and radicular cyst / residual $(4.87 \%)$. The importance of searching for the correct diagnosis of the pathology presented by the patient is so relevant as the choice of methods and techniques to be used in their elimination or control. It is clear that a better and more accurate diagnosis contributes to a well developed treatment.
\end{abstract}

Keywords: Biopsy; Epidemiology; Oral Pathology. 
Dias Neto et al. Levantamento epidemiológico dos diagnósticos histopatológicos de um centro de referência em patologia bucomaxilofacial em um período de 10 anos

\section{INTRODUÇÃO}

O levantamento epidemiológico da casuística das patologias que acometem o complexo bucomaxilofacial é de fundamental importância para a clínica odontológica. ${ }^{(1)}$ Todavia, poucos estudos relacionados a este tema têm sido publicados na literatura, a despeito de sua relevância para a viabilização de programas de saúde envolvendo populações.

Alguns Serviços de Patologia integrados a universidades públicas e privadas têm procurado caracterizar a prevalência de lesões que são obtidas a partir das biópsias realizadas em seus respectivos ambulatórios e centros cirúrgicos. Desta forma, torna-se possível caracterizar o perfil dos pacientes que procuram os serviços especializados destas instituições, levando em conta as suas necessidades de tratamento. ${ }^{(1-7)}$

Vale salientar que, mais do que uma responsabilidade, é dever do cirurgião-dentista detectar quaisquer anormalidades nos tecidos bucais e peribucais. Considerando esta finalidade, um exame clínico acurado, incluindo anamnese minuciosa, deve sempre preceder a uma abordagem terapêutica indicada para cada caso específico. ${ }^{(8)}$ Neste contexto, a biópsia é um procedimento não só necessário, mas imprescindível, para elaboração do plano de tratamento adequado, estabelecendo assim o diagnóstico e prognóstico condizentes com a realidade do paciente. ${ }^{(8)}$

O objetivo deste trabalho foi avaliar a prevalência dos principais tipos de patologias orais que são comumente biopsiados e enviados ao Serviço de Patologia Bucomaxilofacial do Curso de Odontologia da Escola Bahiana de Medicina e Saúde Pública, através da realização de um estudo retrospectivo abrangendo os dados coletados durante os anos de 2001 a 2010.

\section{MATERIAIS E METÓDOS}

Este trabalho foi submetido ao Comitê de Ética em Pesquisa da Escola Bahiana de Medicina e Saúde Pública sob o protocolo 162/09. Foram avaliadas 1095 fichas de biópsias, encaminhadas ao Serviço de Patologia Oral da Escola Bahiana de Medicina e Saúde Pública, durante o período de 2001 a 2010. Tendo como base o diagnóstico histopatológico de cada caso, avaliou-se a prevalência das lesões patológicas, a distribuição geral das amostras quanto ao sexo e idade, a localização das lesões, e a relação entre o diagnóstico clínico e histopatológico. Após a leitura dos laudos, os respectivos diagnósticos foram classificados em diferentes grupos de lesões, de acordo com a sua natureza, conforme ilustra o Quadro 1. 
Dias Neto et al. Levantamento epidemiológico dos diagnósticos histopatológicos de um centro de referência em patologia bucomaxilofacial

Quadro 1 - Distribuição das lesões segundo categorias específicas

1. Infecções bacterianas, micóticas e virais

2. Patologia óssea

3. Patologia de glândulas salivares

4. Lesões hiperplásicas e reativas

5. Neoplasias benignas dos tecidos moles

6. Dermatopatologia

7. Neoplasias malignas dos tecidos moles

8. Cistos e tumores odontogênicos

9. Outros*

* Neste grupo foram incluídos os casos cujas informações clínicas ou amostras disponíveis para o exame anatomo - patológico eram insuficientes para que se chegasse a um diagnóstico final.

\section{RESULTADOS}

Do total de 1095 biópsias, verificou-se que 644 (58,81\%) correspondiam a indivíduos do sexo masculino, enquanto que $451(41,19 \%)$ correspondiam ao sexo feminino. A distribuição etária dos indivíduos biopsiados variou de 03 a 97 anos, com média de idade correspondente a 38 anos. O maior percentual de lesões concentrou-se nas $3^{\circ}, 4^{\circ}, 5^{\circ}$ e $6^{\circ}$ décadas de vida. (Tabelas 1 e 2).

Tabela 1 - Distribuição das lesões segundo o gênero

\begin{tabular}{rcc}
\hline Sexo & N & \% \\
\hline Masculino & 644 & 58,81 \\
Feminino & 451 & 41,19 \\
\hline Total & $\mathbf{1 0 9 5}$ & $\mathbf{1 0 0}$ \\
\hline
\end{tabular}

Tabela 2 - Distribuição dos casos segundo faixa etária

\begin{tabular}{ccc}
\hline Idade & $\mathbf{N}$ & $\mathbf{\%}$ \\
\hline $0-10$ & 52 & 4,75 \\
$11-20$ & 141 & 12,88 \\
$21-30$ & 204 & 18,63 \\
$31-40$ & 175 & 15,98 \\
$41-50$ & 165 & 15,07 \\
$51-60$ & 153 & 13,97 \\
$61-70$ & 97 & 8,86 \\
$71-80$ & 31 & 2,83 \\
\hline
\end{tabular}


Dias Neto et al. Levantamento epidemiológico dos diagnósticos histopatológicos de um centro de referência em patologia bucomaxilofacial em um período de 10 anos

\begin{tabular}{ccc}
\hline $81-100$ & 20 & 1,83 \\
$\mathrm{~S} / \mathrm{R}$ & 57 & 5,21 \\
\hline Total & $\mathbf{1 0 9 5}$ & $\mathbf{1 0 0}$ \\
\hline
\end{tabular}

† S/R corresponde à quantidade de fichas sem identificação da faixa etária.

Tendo em vista os grupos relacionados no Quadro 1, categorizados de acordo com os diferentes tipos de lesões, foram catalogadas as duas patologias mais prevalentes em cada um dos grupos estabelecidos e seu correspondente percentual de prevalência, conforme ilustrado na Tabela 3 .

Tabela 3 - Relação das duas lesões mais prevalentes em cada um dos grupos estabelecidos

\begin{tabular}{|c|c|c|c|}
\hline Grupos & Patologias & $\mathbf{N}$ & $\%$ \\
\hline \multirow[t]{2}{*}{1} & Inflamação crônica granulomatosa & 2 & 50,00 \\
\hline & Paracoccidioidomicose & 1 & 25,25 \\
\hline \multirow[t]{2}{*}{2} & Exostose óssea & 20 & 23,26 \\
\hline & Displasia fibrosa & 17 & 19,77 \\
\hline \multirow[t]{2}{*}{3} & Mucocele & 38 & 48,10 \\
\hline & Adenoma pleomórfico & 14 & 17,72 \\
\hline \multirow[t]{2}{*}{4} & Hiperplasia fibroepitelial & 198 & 48,53 \\
\hline & Inflamação crônica inespecífica & 75 & 18,38 \\
\hline \multirow[t]{2}{*}{5} & Papiloma & 13 & 14,13 \\
\hline & Hemangioma & 12 & 13,04 \\
\hline \multirow[t]{2}{*}{6} & Nevo intradérmico & 16 & 38,09 \\
\hline & Líquen plano & 7 & 16,66 \\
\hline \multirow[t]{3}{*}{7} & Carcinoma escamocelular bem diferenciado & 16 & 37,21 \\
\hline & Carcinoma escamocelular mod.diferenciado & & \\
\hline & & 5 & 11,63 \\
\hline \multirow[t]{2}{*}{8} & Cisto dentígero & 18 & 10,52 \\
\hline & Ameloblastoma & 13 & 7,60 \\
\hline \multirow[t]{2}{*}{9} & Laudo descritivo & 72 & 30,76 \\
\hline & Folículo pericoronário & 36 & 15,38 \\
\hline
\end{tabular}

¥ As porcentagens foram expressas em relação ao número de casos presentes em cada grupo, de acordo com os critérios estabelecidos no Quadro 1.

No grupo das lesões hiperplásicas e reativas foi encontrado o maior número de lesões, dentre as quais a hiperplasia fibroepitelial, que foi a entidade mais representativa, constituiu 
Dias Neto et al. Levantamento epidemiológico dos diagnósticos histopatológicos de um centro de referência em patologia bucomaxilofacial em um período de 10 anos

$48,53 \%$ destas lesões, representando $16,62 \%$ do material total estudado. Já no grupo correspondente às neoplasias malignas de tecidos moles, o carcinoma escamocelular destacou-se como a lesão mais prevalente.

Tabela 4 - Relação das dez lesões mais prevalentes, registradas nos laudos estudados

\begin{tabular}{lcc}
\hline \multicolumn{1}{c}{ Tipos de Patologias } & N & \% \\
\hline Hiperplasia fibroepitelial & 198 & 16,62 \\
Inflamação crônica inespecífica & 75 & 6,30 \\
Cisto radicular/residual & 58 & 4,87 \\
Mucocele & 38 & 3,19 \\
Folículo pericoronário & 36 & 3,02 \\
Granuloma piogênico & 36 & 3,02 \\
Granuloma periapical & 32 & 2,69 \\
Displasia epitelial leve & 21 & 1,76 \\
Hiperceratose & 20 & 1,68 \\
Exostose óssea & 20 & 1,68 \\
Outras & 657 & 55,17 \\
\hline$\quad$ Total & $\mathbf{1 1 9 1}$ & $\mathbf{1 0 0}$ \\
\hline
\end{tabular}

$\S$ A quantidade de lesões catalogadas diferencia-se do número de pacientes, tendo em vista que, em muitos casos, um mesmo indivíduo possuía mais de um tipo de patologia.

Os resultados obtidos em relação aos tipos histológicos encontrados no presente estudo estão expressos na Tabela 4. Entre as lesões catalogadas, verificou-se maior prevalência das seguintes patologias: hiperplasia fibroepitelial (16,62\%); inflamação crônica inespecífica $(6,30 \%)$; cisto radicular/residual $(4,87 \%)$; mucocele $(3,19 \%)$ e folículo pericoronário $(3,02 \%)$.

As infecções bacterianas, micóticas e virais apareceram como o grupo de lesões menos frequentes no estudo. Ressalte-se ainda que, dentro deste grupo, a inflamação crônica granulomatosa constituiu 50\% destas lesões, representando $1,67 \%$ do material total estudado, conforme ilustrado na Tabela 5. Esta tabela relaciona o número total de diagnósticos apresentados, tendo em vista cada um dos grupos descritos no Quadro 1. 
Dias Neto et al. Levantamento epidemiológico dos diagnósticos histopatológicos de um centro de referência em patologia bucomaxilofacial

Tabela 5 - Número e percentual totais descritos em cada grupo

\begin{tabular}{ccc}
\hline Grupo & $\mathbf{N}$ & $\mathbf{\%}$ \\
\hline Lesões hiperplásicas e reativas & 411 & 34,51 \\
Cistos e tumores odontogênicos & 172 & 14,45 \\
Neoplasias benignas dos tecidos moles & 92 & 7,72 \\
Patologia óssea & 89 & 7,47 \\
Patologia de glândulas salivares & 79 & 6,63 \\
Neoplasias malignas de tecidos moles & 46 & 3,86 \\
Dermatopatologia & 42 & 3,53 \\
Infecções bacterianas, micóticas e virais & 04 & 0,34 \\
Outros & 256 & 21,49 \\
\hline Total & $\mathbf{1 1 9 1}$ & $\mathbf{1 0 0}$ \\
\hline
\end{tabular}

Tabela 6 - Comparativo entre diagnósticos - clínico e histopatológico.

\begin{tabular}{ccc}
\hline Comparação & $\mathbf{N}$ & \% \\
\hline $\begin{array}{c}\text { Confirmados } \\
\text { Não confirmados }\end{array}$ & 655 & 59,82 \\
\hline Total & 440 & 40,18 \\
\hline
\end{tabular}

|| Representa a quantidade de diagnósticos clínicos confirmados através de estudos histopatológicos.

A Tabela 6 representa a análise feita entre os diagnósticos clínico e histopatológico, no que tange à sua compatibilidade. Observamos que 59,82\% dos diagnósticos apresentados pelos cirurgiões dentistas foram confirmados através do estudo histopatológico

Tabela 7 - Distribuição dos casos, segundo a localização da lesão

\begin{tabular}{lcc}
\hline \multicolumn{1}{c}{ Localização } & N & \% \\
\hline Mandíbula & 182 & 15,66 \\
Maxila & 141 & 12,13 \\
Lábio & 98 & 8,43 \\
Mucosa jugal & 92 & 7,92 \\
Palato duro/mole & 84 & 7,23 \\
Língua & 68 & 5,85 \\
Extra oral & 54 & 4,65 \\
Rebordo alveolar & 53 & 4,56 \\
\hline
\end{tabular}


Dias Neto et al. Levantamento epidemiológico dos diagnósticos histopatológicos de um centro de referência em patologia bucomaxilofacial

\begin{tabular}{lcc}
\hline Ápice radicular & 52 & 4,48 \\
Gengiva & 46 & 3,96 \\
Região de tuberosidade & 29 & 2,50 \\
Assoalho de boca & 27 & 2,32 \\
Região de sínfise & 24 & 2,07 \\
Fundo de sulco & 16 & 1,38 \\
Região retromolar & 14 & 1,20 \\
S/R & 71 & 6,11 \\
Outros & 111 & 9,55 \\
\hline \multicolumn{2}{c}{ Total } & $\mathbf{1 1 6 2}$ \\
\hline
\end{tabular}

A distribuição de casos, segundo a localização da lesão, está retratada na Tabela 7. Do total de lesões apresentadas, $15,66 \%$ localizaram-se na mandíbula e 12,13\% na maxila. Os sítios menos afetados foram a região de fundo de sulco e retromolar $(1,38 \%$ e 1,20\% respectivamente).

\section{DISCUSSÃO}

O presente estudo procurou identificar as lesões mais prevalentes, bem como a sua localização, tentando agrupá-las através de características histopatológicas. ${ }^{(9,10)}$

A pesquisa abrangeu 1095 pacientes, avaliados entre os anos de 2001 a 2009, sendo que destes, $644(58,81 \%)$ eram do sexo masculino, enquanto que $451(41,19 \%)$ representavam o sexo feminino. Esta relação entre homens e mulheres se contrapõe aos estudos realizados por Torrão et al., ${ }^{(1)}$ Gomez et al., ${ }^{(3)}$ Leonel et al. ${ }^{(5)}$ e Nascimento et al., ${ }^{(7)}$ onde verificou-se maior número de pacientes do sexo feminino.

No que diz respeito à idade dos pacientes, ficou constatada predominância no atendimento de indivíduos entre 21-30 anos, condição semelhante à descrita por Leonel et al., ${ }^{(5)}$ e contrária a que se evidenciou no estudo de Rocha et al., ${ }^{(10)}$ onde a década de vida mais acometida foi a quarta. Nestes termos, a média de idade estabelecida no presente estudo foi de 38 anos, considerando que a faixa etária dos pacientes variou entre os 03 e 97 anos.

De acordo com os grupos estabelecidos, foram catalogadas as lesões mais prevalentes em cada um deles. Do resultado apresentado, ficou estabelecido que a inflamação crônica granulomatosa, a exostose óssea, a mucocele, e assim sucessivamente, foram as lesões mais freqüentes em cada um de seus respectivos grupos. No que se relaciona à hiperplasia 
Dias Neto et al. Levantamento epidemiológico dos diagnósticos histopatológicos de um centro de referência em patologia bucomaxilofacial em um período de 10 anos

fibroepitelial, retratada no grupo de lesões hiperplásicas e reativas, ressalta-se que a mesma foi agrupada juntamente com outras hiperplasias descritas no trabalho, como a hiperplasia fibrosa inflamatória e a hiperplasia epitelial, representando um só grupo de lesões hiperplásicas.

Das patologias diagnosticadas, os resultados coletados indicaram que entre as lesões mais prevalentes destacou-se a hiperplasia fibroepitelial. ${ }^{(11,12)}$ Estes achados ratificam os resultados descritos por Gomez et al., ${ }^{(3)}$ em seu estudo realizado na Faculdade de Odontologia da UFMG, no qual foram avaliadas as biópsias do Serviço de Patologia, utilizando uma metodologia semelhante à descrita neste estudo. Outros autores, a exemplo de Moresco et al., ${ }^{(2)}$ Leonel et al. ${ }^{(5)}$ e Nascimento et al., ${ }^{(7)}$ descreveram resultados semelhantes, evidenciando que, não obstante a diferença regional entre os pacientes avaliados, este tipo de lesão tem sido a que mais acomete a população no país.

O baixo percentual de casos de carcinoma escamocelular bem diferenciado descrito neste estudo retrospectivo (1,34\% dos casos) não deve ser menosprezado. O número de casos (16 em 1191) é relevante, se comparado a outros tipos de patologia, tais como os cistos epidermóide e dentígero, que tiveram prevalência semelhante (17 e 18 casos, respectivamente). Ademais, atenção deve ser dada também para os diagnósticos de lesões consideradas cancerizáveis, que representam patologias que exibem tecidos morfologicamente alterados, a exemplo da displasia epitelial, onde o número de casos foi maior em relação aos carcinomas, sendo 27 em 1.191. Como no estudo de Hosni et al., ${ }^{(13)}$ realizado através de uma avaliação clínica, 13 dos 17.831 prontuários avaliados foram clinicamente diagnosticados como eritroplasia ou leucoplasia salpicada. Estes são tipos de lesões consideradas précancerizáveis, pois em mais de $90 \%$ dos casos já existe displasia. Estudos epidemiológicos referentes a esta modalidade de neoplasia maligna têm sido amplamente descritos na literatura, a exemplo dso realizados por Menezes Filho, ${ }^{(14)}$ Almeida et al., ${ }^{(15)}$ e Borges et al. ${ }^{(16)}$ Salienta-se ainda que o último grupo de autores desenvolveu o projeto Epidemiologia do câncer de boca em laboratório público do Estado do Mato Grosso. Este estudo, realizado através de uma abordagem quantitativa, por meio de uma pesquisa epidemiológica, documental, descrita e transversal, analisou um total de 1.324 laudos emitidos entre janeiro de 2005 e dezembro de 2006, analisando a prevalência de câncer de boca no universo das lesões bucais e as seguintes variáveis: tipo histológico, sexo, idade e procedência dos pacientes (capital ou interior). Realizando uma análise descritiva, o autor concluiu que em dois anos de 
Dias Neto et al. Levantamento epidemiológico dos diagnósticos histopatológicos de um centro de referência em patologia bucomaxilofacial em um período de 10 anos

funcionamento o serviço público de patologia registrou um considerável número de casos de câncer de boca.

No grupo das dermatopatologias, contrapondo o estudo de Nascimento et al., ${ }^{(7)}$ onde o líquen plano, com $42,8 \%$ dos casos, foi a patologia mais encontrada, em nosso trabalho, o nevo intradérmico foi o mais prevalente com 38,09\% dos casos. Este grupo engloba um grande número de condições, em geral de natureza auto-imune ou imunologicamente mediada, que acometem o epitélio da mucosa, trazendo complicações com grande variabilidade clínica. ${ }^{(17)}$

O grupo de cistos e tumores odontogênicos compreendeu 14,45\% do total de biópsias encaminhadas ao serviço, onde a patologia de origem odontogênica mais prevalente foi o cisto dentígero (10,52\%). Este valor difere daqueles relatados por Santos et al. ${ }^{(18)}$ e Regezi et al., ${ }^{(19)}$ que analisaram somente o grupo de tumores odontogênicos, tendo o odontoma sido a patologias mais frequentemente encontrada por eles, com 50,4\% e $67 \%$ dos casos, respectivamente. Já Samuel et al. ${ }^{(20)}$ e Loureiro et al. ${ }^{(21)}$ analisaram os cistos e tumores odontogênicos separadamente. No grupo de cistos odontogênicos, o cisto dentígero foi a patologia mais prevalente, enquanto que, no grupo de neoplasias odontogênicas, o odontoma foi a lesão mais verificada.

No grupo de patologia de glândulas salivares, a mucocele e o adenoma pleomórfico foram às lesões mais prevalentes $(48,10 \%$ e $17,72 \%$, respectivamente). Índice semelhante pode ser também observado nos resultados apresentados por Nascimento et al. ${ }^{(7)}$ e Bettio et al., ${ }^{(6)}$ que avaliaram a prevalência de lesões em glândulas salivares. Todavia, se ambas as lesões forem encaradas dentro do universo de patologias apresentadas, percebe-se que não representaram um percentual significativo. A mucocele, por exemplo, somente foi diagnosticada em 3,19\% dos casos gerais, tendo apresentado percentuais semelhantes em outros trabalhos, como Gomez et al. ${ }^{(3)}$ com $2,92 \%$ dos casos e Bertoja et al. ${ }^{(4)} \operatorname{com} 5,04 \%$ dos casos. Este número reduzido, em relação aos casos gerais, pode ter explicação no tratamento não-cirúrgico desta lesão, aplicado por alguns profissionais.

O cisto radicular aparece como a principal lesão de natureza cística. Observamos semelhança no artigo de Nanami et al. ${ }^{(22)}$ que avalia a prevalência de cistos maxilares e o cisto mais prevalente do levantamento é o radicular com 42,1\% num total de 242.

Embora levantamentos epidemiológicos sejam úteis na estomatologia, determinados cuidados devem ser tomados quando da interpretação dos seus resultados. Devemos lembrar que, embora algumas lesões, como a estomatite aftosa recorrente (Aftas), sejam bastante 
Dias Neto et al. Levantamento epidemiológico dos diagnósticos histopatológicos de um centro de referência em patologia bucomaxilofacial em um período de 10 anos

freqüentes na cavidade bucal, elas não aparecem neste estudo, já que representam processos patológicos em que a biópsia não se constitui um passo semiológico necessário para o diagnóstico final ou para o próprio tratamento. ${ }^{(3)}$ Abordagem semelhante é usualmente adotada para os casos de candidíase eritematosa crônica e estomatites traumáticas associadas ao uso de próteses removíveis. Na maioria destes casos, cuja freqüência em nosso meio é significativa, usa-se o expediente da citologia esfoliativa como exame laboratorial auxiliar para a confirmação do diagnóstico. Por outro lado, há lesões como a estomatite migratória, entre outras, que embora não sendo muito prevalentes, usualmente dispensam a biópsia no seu diagnóstico. ${ }^{(3)}$

Assim os resultados apresentados neste estudo refletem a freqüência apenas daquelas entidades nosológicas que são submetidas à remoção cirúrgica total ou parcial na prática odontológica.

Ainda no que tange às lesões catalogadas e classificadas de acordo com grupos de diagnósticos, tem-se que o grupo de lesões hiperplásicas e reativas foi aquele que apresentou o maior número de casos diagnosticados, assim como observado no trabalho descrito por Gomez et al., (3) onde as lesões hiperplásicas representaram 593 casos, em um universo de 2.566 de lesões. Por outro lado, no estudo de Franco et al., ${ }^{(23)}$ o grupo de lesões ósseas foi aquele que apresentou o maior número de casos diagnosticados, representando 103 diagnósticos em um total de 724 lesões.

Considerando ainda os dados levantados tem-se que, das suspeitas diagnósticas formuladas pelos cirurgiões-dentistas, $59,82 \%$ foram posteriormente confirmadas pelos diagnósticos histopatológicos e 40,18\% tiveram resultados diversos. Se comparados aos resultados apresentados por Reis e Silvany Neto, ${ }^{(24)}$ no seu estudo, que classificou os diagnósticos em 3 tipos (totalmente correto, parcialmente correto e incorreto), a diferença entre os diagnósticos clinico e histopatológico se mostrou muito menos acentuada, já que no trabalho citado foi de apenas $11,6 \%$. Porém, no segundo grupo (resultados diversos) foi constatado que mais da metade deste percentual poderia ser categorizado como "confirmado", uma vez que houve ampla variação de termos que indicavam a mesma patologia. No entanto, apenas os termos idênticos aos descritos na suspeita diagnóstica foram considerados "confirmados".

Em relação à localização das lesões, o presente estudo apresentou maior número de casos na região da mandíbula (15,66\% dos casos apresentados), semelhante aos achados de Nascimento et al., ${ }^{(7)}$ e ao contrário dos dados relacionados por Rocha et al., ${ }^{(10)}$ onde a maior 
Dias Neto et al. Levantamento epidemiológico dos diagnósticos histopatológicos de um centro de referência em patologia bucomaxilofacial em um período de 10 anos

parte das lesões localizou-se na região da mucosa jugal (29,5\% dos casos), e Reis e Silvany Neto, ${ }^{(24)}$ onde a maioria dos casos concentrou-se na maxila $(28,9 \%$ dos casos). Saliente-se, ainda, que o índice de lesões na região da mandíbula dos referidos autores foi de $21,9 \%$, $1,8 \%$ e $16,5 \%$, respectivamente.

\section{CONCLUSÃO}

Os estudos epidemiológicos fornecem uma visão importante para o entendimento da prevalência, da extensão e da severidade das doenças bucais na população. É com base nos resultados destes estudos que os esforços do cirurgião dentista devem concentrar-se, tanto no plano científico e de pesquisas, como no aperfeiçoamento de técnicas de trabalho, escolha dos materiais e maximização dos efeitos do tratamento.

A importância de se buscar o correto diagnóstico da patologia apresentada pelo paciente é tão importante quanto a escolha dos métodos e técnicas a serem utilizadas na sua eliminação, ou controle. É evidente que quanto melhor e mais preciso for o diagnóstico, mais eficiente será o tratamento desenvolvido.

\section{REFERÊNCIAS}

1. Torrão ACR, Rabelo MLM, Soares PL, Nunes RB, Andrade ESS. Levantamento epidemiológico de biópsias da região buco-maxilo-facial encaminhadas ao Laboratório de Patologia Bucal da Faculdade de Odontologia de Pernambuco. Rev Cons Odontol 1999; 2(2):119-125.

2. Moresco FC, Nora Filho MR, Balbinot MA. Levantamento epidemiológico dos diagnósticos histopatológicos da disciplina de Estomatologia da Faculdade de Odontologia da ULBRA - Canoas/RS. Rev Curso Stomatos 2003; 9(17):29-34.

3. Gomez RS, Figueiredo FP, Capistrano HM, Loyola AM. Levantamento das biópsias bucais realizadas na Faculdade de Odontologia da UFMG. Arq Centro Estud Curso Odontol 1992; 29(2):105-113.

4. Bertoja IC, Tomazini JG, Braosi APR, Zielak JC, Reis LFG, Giovanini AF. Prevalência de lesões bucais diagnosticadas pelo Laboratório de Histopatologia do UnicenP. Rev Sul-Bras Odontol 2007; 4(2):41-46.

5. Leonel ECF, Vieira EH, Gabrielli MAC. Análise retrospectiva da incidência, diagnóstico e tratamento das lesões bucais encontradas no Serviço de Cirurgia e Traumatologia Bucomaxilofacial da Faculdade de Odontologia de Araraquara - UNESP. Rev Paul Odontol 2002; 24(3):18-22. 
Dias Neto et al. Levantamento epidemiológico dos diagnósticos histopatológicos de um centro de referência em patologia bucomaxilofacial em um período de 10 anos

6. Bettio A, Salgado G, Azevedo-Alanis LR, Machado MAN, Grégio AMT, Lima AAS. Prevalência das Lesões de Glândulas Salivares em Laudos Histopatológicos do Laboratório de Patologia Experimental da PUCPR no período de 1999-2008. Rev Sul-Bras Odontol 2009; 6(3):231-236.

7. Nascimento GJF, Paraíso DP, Góes PSA, Sobral APV. Estudo epidemiológico de 2.147 casos de lesões bucomaxilo-faciais. Rev Bras Patol Oral 2005; 4(2):01-11.

8. Barbosa RPS, Paiva MDEB, Rodrigues TLC, Rodrigues FG. Valorizando a biópsia na clínica odontológica. Arq Odontol 2005; 41(4):318-328.

9. Cruz MCFN, Almeida KGB, Lopes FF, Bastos EG, Freitas RA. Levantamento das biópsias da cavidade oral realizadas no hospital universitário - Unidade Presidente Dutra / UFMA, da Cidade de São Luís - MA, no período de 1992 a 2002. Rev Bras Patol Oral 2005; 4(3):185188.

10. Rocha DAP, Oliveira LMM, Souza LB. Neoplasias benignas da cavidade oral: estudo epidemiológico de 21 anos (1982 a 2002). Rev Odontol UNICID 2006; 18(1):53-60.

11. Neville BW, Damm DD, Allen CM, Bouquot JE. Patologia oral e maxilofacial. Rio de Janeiro: Guanabara Koogan; 2004. p. 419-475.

12. Boraks S. Diagnóstico bucal. São Paulo: Artes Médicas; 2001. p. 326-346.

13. Hosni ES, Salum FG, Cherubini K, Yurgel LS, Figueiredo MAS. Eritoplasia e leucoeritoplasia oral: análise retrospectiva de 13 casos. Rev Bras Otorrinolaringol 2009; 75(2):295-299.

14. Menezes Filho JF. Incidências de neoplasias BMF. RGO (Porto Alegre) 1991; 39(6):428430 .

15. Almeida OP, Silva, CRV, Saiki P. Levantamento de lesões bucais. RGO (Porto Alegre) $1987 ; 35(6): 471-473$

16. Borges FT, Garbin CAS, Carvalhosa AA, Castro PHS, Hidalgo LRC. Cad Saúde Pública 2008; 24(9):1977-1982.

17. Neville BW, Damm DD, Allen CM, Bouquot JE. Patologia oral e maxilofacial. Rio de Janeiro: Guanabara Koogan; 2004. p. 617-675.

18. Santos JN, Pinto LP, Figueredo CRLV, Souza LB. Odontogenic tumors: Analysis of 127 Cases. Pesqui Odontol Bras 2001; 15(4): 308-313.

19. Regezi JA, Kerr DA, Courtney RM. Odontogenic tumors: analysis of 706 cases. J Oral Surg 1978; 36(10):771-778. 
Dias Neto et al. Levantamento epidemiológico dos diagnósticos histopatológicos de um centro de referência em patologia bucomaxilofacial em um período de 10 anos

20. Samuel MMS, Figueiredo MAZ, Lorandi CS, Yurgel LS. Levantamento de diagnósticos histopatológicos de um Laboratório de Patologia Bucomaxilofacial, em um período de 10 anos. Rev Odonto Ciênc 1989; 4(7):73-91.

21. Loureiro MS, Duarte R, Figueiredo MAZ, Lorandi CS, Yurgel L. Levantamento epidemiológico dos diagnósticos histopatológicos de um Laboratório de Patologia Bucomaxilofacial em um Período de 18 anos. Rev Odonto Ciênc 1997; 12(24):117-130.

22. Nanami R, Sampaio C, Olivete J, Pizzatto E, Moresca R, Giovanini AF. Prevalência de cistos maxilares diagnosticados em um Centro de Referência Brasileiro. Rev Sul-Bras Odontol 2009; 6(2):143-146.

23. Franco BM, Bonan PRF, Reis C, Barbosa DR, Martelli Júnior H. Lesões buco-faciais apresentadas durante as "Jornadas Mineiras de Estomatologia" entre 1995 a 2006. Arq Odontol 2006; 42(2):133-139.

24. Reis SRA, Silvany AMN. Concordância entre o Diagnóstico clínico e histopatológico em lesões da cavidade oral. Rev Odonto Ciênc 1996; 11(21):183-195. 